UTAP-352

astro-ph/9912463

\title{
Kinetically Driven Quintessence
}

\author{
Takeshi Chiba*, Takahiro Okabe ${ }^{\dagger}$, and Masahide Yamaguchi ${ }^{\ddagger}$ \\ Department of Physics, University of Tokyo, Tokyo 113-0033, Japan
}

(November 12, 1999)

\begin{abstract}
Recently, a novel class of models for inflation has been found in which the inflationary dynamics is driven solely by (non-canonical) kinetic terms rather than by potential terms. As an obvious extension, we show that a scalar field with non-canonical kinetic terms alone behaves like an energy component which is time-varying and has negative pressure presently, i.e. quintessence. We present a model which has a constant equation of state, that is, a "kinetic" counterpart of the Ratra-Peebles model of a quintessence field with a potential term. We make clear the structure of the phase plane and show that the quintessential solution is a late-time attractor. We also give a model for the "phantom" component which has an equation of state with $w=p / \rho<-1$.
\end{abstract}

PACS numbers: $98.80 . \mathrm{Cq}$

*Electronic address: chiba@utap.phys.s.u-tokyo.ac.jp, JSPS research fellow.

$\dagger$ Electronic address: okabe@utap.phys.s.u-tokyo.ac.jp

‡Electronic address: gucci@utap.phys.s.u-tokyo.ac.jp, JSPS research fellow. 


\section{INTRODUCTION}

Recent indirect or direct observations suggest that the Universe is currently dominated by an energy component with negative pressure [1 4 . One possibility for such a component is the cosmological constant. Another possibility is dynamical vacuum energy or quintessence, a temporary decreasing and spatially inhomogeneous component with negative pressure [5-18]. Only recently, a more radical candidate (called "phantom" component) has been proposed which is "growing" in time [19].

Two problems arise from such a vacuum energy. The first is the fine-tuning problem: the vacuum energy density of order $\sim 10^{-47} \mathrm{GeV}^{4}$ requires the introduction of a new mass scale about 14 orders of magnitude smaller than the electroweak scale. The second is the coincidence problem: the conditions in the early universe have to be set very carefully in order for the energy density of the vacuum and that of the matter to be comparable today. These problems are degenerate for the cosmological constant, however, they are separated in quintessence. A class of quintessence can avoid the coincidence problem by means of the attractor solution [7, 13]. It is shown that the quintessence field approaches a common evolutionary track for a very wide range of initial conditions, so that the cosmology is extremely insensitive to the initial conditions.

Usually the quintessence field is modeled by a scalar field with a canonical kinetic term and a potential term. However, we show that a scalar field with solely kinetic terms can (even without potential terms), albeit they are non-canonical, mimic such a (canonical) quintessence field. Our model is a natural extension of the kinetically driven inflation model proposed recently [20]. A mechanism is proposed by which a dilaton remains massless [21]. Assuming universality of the dilaton coupling functions, it has been shown that the dilaton evolves cosmologically towards values where it decouples from matter (so called "Least Coupling Principle") [21].

Unlike the usual potentially driven quintessence model which automatically satisfies the weak energy condition, allowing non-canonical kinetic terms enables us to model the missing energy component which violates even the weak energy condition (so called "phantom field" [19]). Only recently, Caldwell draw our attention to consider more general equation of state with $w=p / \rho<-1$ [19]. As he noted, since such a "phantom" equation-of-state cannot be achieved with a canonical Lagrangian and Einstein gravity, considering the phantom field requires some extension: either (i) non-canonical Lagrangian or (ii) non-Einstein gravity (or both). The latter possibility seems unlikely since the deviation from general relativity at the present time is strongly constrained by the solar system experiments [22]. Our attempt is minimal one: non-canonical kinetic terms without a potential term. We intend to develop a more general study by including potential terms as well in the near future.

The organization of the paper is as follows. In Sec.II, we present our model. In Sec.III, we start to show the existence of a scaling solution with a constant equation of state, $-1<w<0$. Then we show that the scaling solution is a late-time attractor by means of linear and numerical analyses. In Sec.IV], we give a model which has a scaling solution with a constant equation of state of $w<-1$, and show that the scaling solution is a late-time attractor. In Sec. $\mathrm{M}$, we make a comment on the possibility of reconstructing Lagrangian through observational data. Sec. $\nabla]$ is devoted to summary. 


\section{BASICS}

We consider the following action of a single scalar field $\phi$ minimally coupled with gravity:

$$
S=\int d^{4} x \sqrt{-g}\left(\frac{1}{2 \kappa^{2}} R+p(\phi, \nabla \phi)\right)+S_{B},
$$

where $\kappa^{2} \equiv 8 \pi G$ and $S_{B}$ denotes the action of the background matter and/or radiation. Following [20], for simplicity, we only consider Lagrangians which depend only on the scalar field $\phi$ and its derivative squared円

$$
X \equiv-\frac{1}{2} \nabla^{\mu} \phi \nabla_{\mu} \phi
$$

For the general action of the scalar field (2.1), the field equations are given by

$$
R_{\mu \nu}-\frac{1}{2} g_{\mu \nu} R=\kappa^{2}\left(\frac{\partial p(\phi, X)}{\partial X} \nabla_{\mu} \phi \nabla_{\nu} \phi+p(\phi, X) g_{\mu \nu}+T_{\mu \nu}^{B}\right)
$$

where $T_{\mu \nu}^{B}$ denotes the energy-momentum tensor of the background. Eq.(2.3) shows that $p(\phi, X)$ in the action (2.1) actually corresponds to the "pressure", $p_{\phi}$, of the scalar field [20], while the energy density, $\rho_{\phi}$, is given by $\rho_{\phi}=2 X \partial p / \partial X-p$. Hence the extrema of $p(\phi, X)$ with respect to $X$ correspond to the same equation of state as that of a cosmological constant: $\rho_{\phi}+p_{\phi}=2 X \partial p / \partial X=0$.

We assume that the universe is described by a flat homogeneous and isotropic universe model with the scale factor $a$. The time coordinate is so normalized that $a=1$ at present. The field equations are then

$$
\begin{aligned}
& H^{2}:=\left(\frac{\dot{a}}{a}\right)^{2}=\frac{\kappa^{2}}{3}\left(\rho_{B}+\rho_{\phi}\right)=\frac{\kappa^{2}}{3}\left(\rho_{B}+2 X \frac{\partial p}{\partial X}-p\right), \\
& \frac{\ddot{a}}{a}=-\frac{\kappa^{2}}{6}\left(\rho_{B}+3 p_{B}+\rho_{\phi}+3 p_{\phi}\right), \\
& \dot{\rho}_{B}=-3 H\left(\rho_{B}+p_{B}\right)=:-3 H\left(1+w_{B}\right) \rho_{B}, \\
& \ddot{\phi}\left(\frac{\partial p}{\partial X}+\dot{\phi}^{2} \frac{\partial^{2} p}{\partial X^{2}}\right)+3 H \frac{\partial p}{\partial X} \dot{\phi}+\frac{\partial^{2} p}{\partial X \partial \phi} \dot{\phi}^{2}-\frac{\partial p}{\partial \phi}=0
\end{aligned}
$$

where $\rho_{B}$ and $p_{B}$ are the energy density and the pressure of the background matter and/or radiation, respectively.

Since we only consider kinetic terms, we must impose that the function $p(\phi, X)$ vanishes when $X \rightarrow 0$. Near $X=0$, a generic Lagrangian may be expanded as

$$
p(\phi, X)=K(\phi) X+L(\phi) X^{2}+\cdots .
$$

\footnotetext{
${ }^{1}$ We use the metric signature $(-+++)$.

${ }^{2}$ This amounts to assuming some resolution of the cosmological constant problem. The situation is the same as assuming the minimum of the potential energy is zero in the (canonical) quintessence field with a potential term.
} 


\section{POWER-LAW KINETIC QUINTESSENCE}

To see the effect of non-canonical kinetic terms in a concrete matter, in this section, we shall concentrate on the simplest Lagrangian containing only $\dot{\phi}^{2}$ and $\dot{\phi}^{4}$ terms, namely

$$
p(\phi, X)=K(\phi) X+L(\phi) X^{2} .
$$

In order to realize a model with negative pressure, $K$ and/or $L$ should be negative (note that $X \geq 0$ ). However, for the positivity of $\rho_{\phi}$ for large $X$, we assume that $L$ is always

positive. We thus consider the case of $K<0$. By redefining the scalar field and working with new field variable such that

$$
\phi_{\text {new }}=\int^{\phi_{\text {old }}} d \phi \frac{L(\phi)^{1 / 2}}{|K(\phi)|^{1 / 2}}
$$

we rewrite Eq.(3.1) as

$$
p(\phi, X)=f(\phi)\left(-X+X^{2}\right)
$$

where $\phi \equiv \phi_{\text {new }}, X \equiv X_{\text {new }}=(L /|K|) X_{\text {old }}$ and $f(\phi) \equiv K^{2}\left(\phi_{\text {old }}\right) / L\left(\phi_{\text {old }}\right)$. We may regard Eq.(3.3) as the basic Lagrangian. Then the pressure, $p_{Q}$, and energy density, $\rho_{Q}$, of the quintessence is given by

$$
\begin{aligned}
& p_{Q}=f(\phi)\left(-X+X^{2}\right), \\
& \rho_{Q}=2 X \frac{\partial p}{\partial X}-p=f(\phi)\left(-X+3 X^{2}\right) .
\end{aligned}
$$

\section{A. scaling solution}

We look for scaling solutions which keep $w_{Q} \equiv p_{Q} / \rho_{Q}$ constant. Then from Eqs.(3.4) and (3.5), $X$ is also found to be constant:

$$
X=\frac{1-w_{Q}}{1-3 w_{Q}}
$$

During the matter or radiation dominated epoch $\left(\rho_{B} \gg \rho_{Q}\right)$, Eq.(2.7) becomes

$$
\dot{\rho}_{Q}=-\frac{2}{t\left(1+w_{B}\right)}\left(1+w_{Q}\right) \rho_{Q}
$$

Substituting Eqs.(3.5) and (3.6) into the above equation, we thus obtain

$$
f(\phi) \propto\left(\phi-\phi_{*}\right)^{-2\left(1+w_{Q}\right) /\left(1+w_{B}\right)},
$$

where $\phi_{*}$ is a constant. For simplicity, we henceforth choose $\phi_{*}=0$.

To summarize, for the scalar field model with the constant equation of state, $w_{Q}$, during the matter or radiation dominated epoch, the function $f(\phi)$ should take the form of Eq.(3.8). Conversely, if the function $f(\phi)$ is given by 


$$
f(\phi) \propto \phi^{-\alpha}
$$

then there exists a scaling solution such that the equation of state is characterized by

$$
w_{Q}=\frac{\left(1+w_{B}\right) \alpha}{2}-1
$$

Hence if we require that $w_{Q}<0$ during the matter dominated epoch, then the exponent $\alpha$ should satisfy

$$
\alpha<2 \text {. }
$$

Note that the weak energy condition $\left(w_{Q} \geq-1\right)$ can be violated if $\alpha<0$. The stability against perturbations is signified by the "speed of sound" defined by 20]

$$
c_{s}^{2}=\frac{p_{Q, X}}{\rho_{Q, X}}=\frac{p_{, X}}{p_{, X}+2 X p_{, X X}} .
$$

For the quartic model Eq.(3.4), one finds $c_{s}^{2}=\left(1+w_{Q}\right) /\left(5-3 w_{Q}\right)$, and the model is unstable for perturbations on all length scales if the weak energy condition is violated: $w_{Q}<-1$. One may wonder this is always the case. However, this is not so. In fact, we can consider a more general Lagrangian of the form 20]

$$
p(\phi, X)=f(\phi) g(X),
$$

where $g(X)$ is an arbitrary function of $X$. One can show the equation of motion Eq.(3.7) has a solution $X_{0}=$ const. with the function $f(\phi)$ being the same form as Eq.(3.8) if $X_{0}$ satisfies

$$
\left.2 \frac{\partial \ln g}{\partial \ln X}\right|_{X=X_{0}}=\frac{1+w_{Q}}{w_{Q}}
$$

Note that Eq.(3.12) involves the second derivative $g_{, X X}\left(X_{0}\right)$. Therefore, one can always arrange it so that $c_{s}^{2}>0$ for stability. This may be a concrete realization of the "phantom" field (or "growing lambda") using the non-canonical Lagrangian 19. An example of such a phantom field will be considered in Sec.IV. In this section, we shall limit ourselves to the case of $0<\alpha<2$ so that $-1<w_{Q}<0$.

\section{B. attractor structure}

In the matter or radiation dominated universe, there exists the scaling solution for the kinetic Lagrangian. Here, we show that this solution is an attractor of the equation of motion for the scalar field. When $\rho_{B} \gg \rho_{Q}$, Eq.(2.7) becomes

$$
\ddot{\phi}\left(1-3 \dot{\phi}^{2}\right)+\frac{2}{t\left(1+w_{B}\right)}\left(1-\dot{\phi}^{2}\right) \dot{\phi}+\frac{f^{\prime}}{4 f}\left(2-3 \dot{\phi}^{2}\right) \dot{\phi}^{2}=0 \text {. }
$$

Since this equation has reflection symmetry $\phi \leftrightarrow-\phi$, we mainly consider the case of $\phi>0$.

Following [7,25], we make the change of variables as 


$$
\begin{aligned}
\tau & \equiv \ln t \\
u & \equiv \frac{\phi}{\phi_{s}}
\end{aligned}
$$

where the scaling solution, $\phi_{s}$, is given by

$$
\phi_{s}=\sqrt{\frac{2\left(1-w_{Q}\right)}{1-3 w_{Q}}} t=: \xi_{s} t
$$

With these changes, Eq.(3.15) becomes

$$
\begin{aligned}
u^{\prime}= & v, \\
v^{\prime}= & -v+\frac{1}{1-3 \xi_{s}^{2}(v+u)^{2}} \\
& \times\left[\frac{2}{1+w_{B}}\left\{-(v+u)+\xi_{s}^{2}(v+u)^{3}\right\}-\frac{\alpha}{4 u}\left\{-2(v+u)^{2}+3 \xi_{s}^{2}(v+u)^{4}\right\}\right],
\end{aligned}
$$

where' denotes the derivative with respect to $\tau$. Then one can find three critical points; $(u, v)=(0,0),(1,0)$ and $(-1,0)$. The $(-1,0)$ critical point corresponds to the scaling solution with negative amplitude, while the $(0,0)$ critical point is a trivial one in which $X=0$.

In order to study the stability near the critical points, we use linear analysis [7, 25]. Perturbing about the scaling solution $(u, v)=(1+\delta u, 0+\delta v)$ and keeping only the terms linear in $\delta u$ and $\delta v$, Eqs.(3.19) and (3.20) become

$$
\begin{aligned}
& \delta u^{\prime}=\delta v \\
& \delta v^{\prime}=-\delta v+\frac{2 w_{Q}}{1+w_{B}}(\delta u+\delta v)-\frac{1+w_{Q}}{2\left(1+w_{B}\right)} \frac{2-3 \xi_{s}^{2}}{1-3 \xi_{s}^{2}} \delta u .
\end{aligned}
$$

Then the eigenvalues of small perturbations are given by

$$
\lambda_{s}^{ \pm}=\frac{2 w_{Q}-1-w_{B} \pm \sqrt{\left(2 w_{Q}-1-w_{B}\right)^{2}+8\left(1+w_{B}\right)\left\{w_{Q}-\left(1+w_{Q}\right) /\left(5-3 w_{Q}\right)\right\}}}{2\left(1+w_{B}\right)} .
$$

The necessary and sufficient condition for stability is that the real part of the eigenvalues be negative. Note that because $-1<w_{Q}<0$, the second term under the square root is always negative. Then the condition for the stability is just $2 w_{Q}-1-w_{B}<0$. Hence the $(1,0)$ critical point corresponding to the scaling solution is stable. In a similar way, the eigenvalues of small perturbations near the trivial solution is given by

$$
\lambda_{t}^{ \pm}=\frac{2 w_{Q}-1-w_{B} \pm \sqrt{\left(2 w_{Q}-1-w_{B}\right)^{2}+4\left(1+w_{B}\right)\left(w_{Q}-1\right)}}{2\left(1+w_{B}\right)} .
$$

Then the $(0,0)$ critical point is also stable. 


\section{C. numerical analysis}

We have shown that the scaling solution is stable for small perturbations. In order to analyze the phase plane, we solve Eqs.(3.19) and (3.20) numerically. The phase plane is shown in FIG. 1 for the case of $\alpha=1$ and $w_{B}=0$. This figure shows the attractor structure of the scaling solutions and the trivial solution: there are trajectories which converge on these solutions asymptotically. The boundaries between phase flow, lines (1) and (2) in FIG. 1, correspond to the lines where Eq.(3.20) is singular:

$$
v=-u \pm \sqrt{\frac{1-3 w_{Q}}{6\left(1-w_{Q}\right)}} .
$$

Eq.(3.20) is also singular on $u=0$ except for

$$
v=v_{ \pm}:= \pm \sqrt{\frac{1-3 w_{Q}}{3\left(1-w_{Q}\right)}} .
$$

The trajectories in the region $v<-u+\sqrt{\left(1-3 w_{Q}\right) / 6\left(1-w_{Q}\right)}$ and $u>0$ approach and pass through the point $(u, v)=\left(0, v_{-}\right)$, then converge on the scaling solution with negative amplitude. From Eqs.(3.12), the requirement for stability against perturbations is not satisfied in the region

$$
\frac{1-3 w_{Q}}{6\left(1-w_{Q}\right)}<(u+v)^{2}<\frac{1-3 w_{Q}}{2\left(1-w_{Q}\right)} .
$$

These regions correspond to the shaded ones in FIG. 1.

The cosmological evolution of the scalar field is obtained by solving Eqs.(2.5) and (2.7) numerically. The initial conditions for the scale factor are so chosen that the Friedmann equation (2.4) is satisfied. Those for the scalar field are chosen in the region $u>0$ and $v>$ $-u+\sqrt{\left(1-3 w_{Q}\right) / 2\left(1-w_{Q}\right)}$ so that $c_{s}^{2}>0$ initially. In FIG. 2, we show the time evolution of energy densities of radiation, matter and quintessence field for various initial conditions. The present density parameter of the $i$-th component $\Omega_{i}$ is defined by $\Omega_{i, 0}=\kappa^{2} \rho_{i}^{0} /\left(3 H_{0}^{2}\right)$. We choose $\Omega_{M, 0}=0.25$ and set $\alpha=1$. The figure shows that for a very wide range of initial conditions, the energy density of the quintessence field converges on a common evolutionary track.

\section{D. mass scale}

In the Ratra-Peebles model of the quintessence field driven by a potential term, one can choose a parameter with mass dimension in the potential term to be a typical particle physics scale [13]. We introduce a parameter with mass dimension in the kinetic Lagrangian as

$$
f(\phi)=\frac{M^{4-\alpha}}{\phi^{\alpha}}
$$


then we fix this parameter by requiring that the scalar field is beginning to dominate the energy density of the universe today $\left(\rho_{\text {crit }}^{0}\right.$ is the present critical density and $\phi_{0}$ is the present value of the scalar field)

$$
\rho_{Q}^{0}=f\left(\phi_{0}\right)\left(-X+3 X^{2}\right) \simeq \rho_{\text {crit }}^{0},
$$

and that the scalar field has already reached the attractor solution

$$
\left.\frac{f^{\prime}}{f}\right|_{\phi=\phi_{0}} \simeq \frac{1}{\phi_{0}} \simeq H_{0} .
$$

The last condition is obtained from Eq.(3.15). These conditions fix the mass parameter and the present value of the scalar field as

$$
\begin{aligned}
M & \sim 10^{(43 \alpha-48) /(4-\alpha)}[\mathrm{GeV}], \\
\phi_{0} & \sim 10^{43}\left[\mathrm{GeV}^{-1}\right],
\end{aligned}
$$

respectively. $M$ could be larger than $\mathrm{TeV}$ scale when $\alpha \gtrsim 1.3$.

\section{PHANTOM FIELD}

In this section, we construct a model of a power-law phantom field which is recently proposed as an alternative to the dark energy component [19]. In fact, in Ref. [19], a scalar field with a kinetic term of inverted sign is introduced as a toy model of such a component: the Lagrangian density is given by $\mathcal{L}_{P}=+\frac{1}{2} \nabla_{\mu} \phi \nabla^{\mu} \phi-V(\phi)$. The concern is the tachyonic instability for $\phi$. However, as shown in [19], as long as $V_{, \phi \phi}$ is negative, such an instability is not developed. For example, for a constant equation of state, one can show $V_{, \phi \phi}=\frac{3}{2}\left(1-w_{P}\right)\left[\dot{H}-\frac{3}{2} H^{2}\left(1+w_{P}\right)\right]$, thus it is negative as long as $-2<w_{P}<-1$. Our model will not suffer from such a restriction even for a constant equation of state.

\section{A. scaling solution}

In the previous section, we have mentioned that a general Lagrangian of the form Eq.(3.13), $p(\phi, X)=f(\phi) g(X)$, with the function $f(\phi)$ of the form Eq.(3.9) has scaling solutions with $X=$ constant. From Eq. (3.10), the equation of state is characterized by

$$
w_{P} \equiv \frac{p_{P}}{\rho_{P}}=\frac{\left(1+w_{B}\right) \alpha}{2}-1
$$

then the phantom field which violates the weak energy condition, $w_{P}<-1$, corresponds to $\alpha<0$.

We determine the function $g(X)$ which has a phantom solution. We impose the following conditions on $g(X)$ other than $g(X) \rightarrow 0$ for $X \rightarrow 0$ (vacuum triviality) and $g(X)>0$ for $X \rightarrow \infty$ (positivity of the energy density for large $X$ ): (i) the positivity of the energy density $\rho_{P}=f\left(2 X g_{, X}-g\right)>0$ because we are considering the missing energy component; (ii) the 
violation of the weak energy condition, $\rho_{P}+p_{P}=2 f X g_{, X}<0$; (iii) the stability against perturbations, $c_{s}^{2}=g_{, X} /\left(g_{, X}+2 X g_{, X X}\right)>0$.

In terms of $\dot{\phi}$ (so that $X=\dot{\phi}^{2} / 2$ ) these conditions are rewritten as: (i') $g, \dot{\phi} \phi-g>0$, which is geometrically interpreted as the intersection of the tangent to the curve $g=g(\dot{\phi})$ at the point $\dot{\phi}$ with the vertical axis is negative; (ii') $\dot{\phi} g_{, \dot{\phi}}<0$; (iii') $g_{, \dot{\phi}} /\left(\dot{\phi} g_{, \dot{\phi} \phi}\right)>0$. Thus, for $\dot{\phi}>0, g(\dot{\phi})$ should satisfy $g_{, \dot{\phi}}<0$ and $g_{, \dot{\phi} \dot{\phi}}<0$. Therefore, $g(\dot{\phi})$ must have at least three extrema for $\dot{\phi}>0$ and hence be an eighth-order function of $\dot{\phi}$. We parameterize $g(\dot{\phi})$ asp]:

$$
\begin{aligned}
g(\dot{\phi}) & =\int_{0}^{\dot{\phi}} \dot{\phi}\left(\dot{\phi}^{2}-b^{2}\right)\left(\dot{\phi}^{2}-c^{2}\right)\left(\dot{\phi}^{2}-d^{2}\right) d \dot{\phi} \\
& =: \frac{1}{8} \dot{\phi}^{8}-\frac{1}{6} A \dot{\phi}^{6}+\frac{1}{4} B \dot{\phi}^{4}-\frac{1}{2} C \dot{\phi}^{2},
\end{aligned}
$$

where $b, c$ and $d$ are constants corresponding to the extrema of $g(\dot{\phi})$ as shown in FIG. 3. A sketch of $g(\dot{\phi})$ which satisfies the above conditions (i'), (ii") and (iii') is shown there. In the shaded regions, $c_{s}^{2}<0$ so that the solution is unstable for perturbations on all length scale. From the above equation, the scaling solutions satisfy

$$
w_{P}=\frac{C \dot{\phi}^{2} / 2-B \dot{\phi}^{4} / 4+A \dot{\phi}^{6} / 6-\dot{\phi}^{8} / 8}{C \dot{\phi}^{2} / 2-3 B \dot{\phi}^{4} / 4+5 A \dot{\phi}^{6} / 6-7 \dot{\phi}^{8} / 8} .
$$

Then there are six scaling solutions at most.

\section{B. attractor structure}

We show that the phantom solution is an attractor of the equation of motion for the scalar field by means of the linear analysis employed in the previous section. From Eq.(4.2), the equation of motion (2.7) becomes

$$
\begin{aligned}
& \ddot{\phi}\left(C-3 B \dot{\phi}^{2}+5 A \dot{\phi}^{4}-7 \dot{\phi}^{6}\right)+\frac{2}{t\left(1+w_{B}\right)}\left(C-B \dot{\phi}^{2}+A \dot{\phi}^{4}-\dot{\phi}^{6}\right) \dot{\phi} \\
& +\frac{f^{\prime}}{f}\left(\frac{1}{2} C \dot{\phi}^{2}-\frac{3}{4} B \dot{\phi}^{4}+\frac{5}{6} A \dot{\phi}^{6}-\frac{7}{8} \dot{\phi}^{8}\right)=0,
\end{aligned}
$$

when $\rho_{B} \gg \rho_{P}$. We make the change of variables as (3.16) and

$$
u \equiv \frac{\phi}{\phi_{P}}=: \frac{\phi}{\xi_{P} t},
$$

where $\xi_{P}$ is determined by one of the solutions of Eq.(4.3) corresponding to the phantom solution. With these changes, Eq.(4.4) becomes

3 We take negative sign for the coefficient of $\dot{\phi}^{2}$ as a minimal extension of the quartic model Eq.(3.3). If we take positive sign, then the polynomial should be at least of tenth-order. 


$$
\begin{aligned}
u^{\prime}= & v \\
v^{\prime}= & -v+\frac{1}{C-3 B \xi_{P}^{2}(v+u)^{2}+5 A \xi_{P}^{4}(v+u)^{4}-7 \xi_{P}^{6}(v+u)^{6}} \\
& \times\left[\frac{2}{1+w_{B}}\left\{-C(v+u)+B \xi_{P}^{2}(v+u)^{3}-A \xi_{P}^{4}(v+u)^{5}+\xi_{P}^{6}(v+u)^{7}\right\}\right. \\
& \left.-\frac{\alpha}{u}\left\{-\frac{1}{2} C(v+u)^{2}+\frac{3}{4} B \xi_{P}^{2}(v+u)^{4}-\frac{5}{6} A \xi_{P}^{4}(v+u)^{6}+\frac{7}{8} \xi_{P}^{6}(v+u)^{8}\right\}\right],
\end{aligned}
$$

where ' denotes the derivative with respect to $\tau$. Then one can find seven critical points at most; $(u, v)=(0,0),( \pm 1,0)$ and others.

Perturbing about the phantom solution $(u, v)=(1+\delta u, 0+\delta v)$ and keeping only the terms linear in $\delta u$ and $\delta v$, Eqs.(4.6) and (4.7) become

$$
\begin{aligned}
& \delta u^{\prime}=\delta v, \\
& \delta v^{\prime}=-\delta v+\frac{2 w_{P}}{1+w_{B}}(\delta u+\delta v)-\frac{2\left(1+w_{P}\right)}{1+w_{B}} S \delta u,
\end{aligned}
$$

where

$$
\begin{aligned}
S & =\frac{C / 2-3 B \xi_{P}^{2} / 4+5 A \xi_{P}^{4} / 6-7 \xi_{p}^{6} / 8}{C-3 B \xi_{P}^{2}+5 A \xi_{P}^{4}-7 \xi_{p}^{6}} \\
& =\left.\frac{1}{\xi_{P}^{2}} \frac{\dot{\phi} g_{, \dot{\phi}}-g}{g_{, \dot{\phi} \dot{\phi}}}\right|_{\dot{\phi}=\xi_{P}} .
\end{aligned}
$$

Then the eigenvalues of small perturbations are given by

$$
\lambda_{P}^{ \pm}=\frac{2 w_{Q}-1-w_{B} \pm \sqrt{\left(2 w_{P}-1-w_{B}\right)^{2}+8\left(1+w_{B}\right)\left\{w_{P}-\left(1+w_{P}\right) S\right\}}}{2\left(1+w_{B}\right)} .
$$

Since we impose $\rho_{P}>0$ and $g_{, \dot{\phi} \dot{\phi}}<0$ on the phantom solution, $S<0$. Then the second term under the square root is negative. Therefore the $(1,0)$ critical point corresponding to the phantom solution is stable. In a similar way, the eigenvalues of small perturbations near the trivial solution $(0,0)$ is given by Eq.(3.24) but $w_{Q}$ is replaced with $w_{P}$ since the phase structure around the trivial solution is determined by the lowest kinetic term. The trivial solution is also stable.

\section{C. numerical analysis}

In FIG. 4 , the phase plane for the case of $\alpha=-1$ and $w_{B}=0$ is shown by solving Eqs.4.6) and (4.7) numerically. We choose $b^{2}=1 / 2, c^{2}=1$ and $d^{2}=2$ (or $A=7 / 2, B=$ $7 / 2, C=1)$. There are one trivial solution and six scaling solutions. The shaded regions correspond to those in FIG. 3, $c_{s}^{2}<0$. Eq.(4.7) is singular on the dashed lines except for the points represented by cross. The boundaries between phase flow correspond to these singular lines. The trajectories in the region above line (1) and $u>0$ converge on

the scaling solutions. In the region between line (1) and (2), they converge on the trivial 
solution. In the region below line $(2)$ and $u>0$, trajectory approaches a point on the boundary asymptotically.

The cosmological evolution of the scalar field is obtained by solving Eqs.(2.5) and (2.7) numerically. In FIG. 5, we show the time evolution of energy densities of radiation, matter and phantom field for various initial conditions. We choose $\Omega_{M, 0}=0.25$ and set $\alpha=-1$. Since the scaling solution is growing in time and bounded by the instability regions (in which $w>-1$ ) as shown in FIG. 3, the initial energy density should be chosen to be below the present energy scale. Hence, there remains a severe fine-tuning problem of the initial conditions. We expect that including potential terms or introducing additional fields may allow initially decaying solutions. We note, however, that the condition (ii), $\rho+p=2 f X g_{, X}<0$, remains unchanged even if we include potential terms.

\section{RECONSTRUCTING $P(\phi, X)$}

It has been shown that the effective potential of a scalar field with a canonical kinetic term can be determined by using the magnitude-redshift relation of distant type Ia supernovae [23,24. In a similar way, we consider the possibility of reconstructing $p$ as a function of $\phi$ and $X$ through observational data. The pressure and density of the quintessence or phantom component can be written in terms of the coordinate distance to redshift $z, r(z)$ (which is related to the luminosity distance, $\left.d_{L}(z)=(1+z) r(z)\right)$ as

$$
\begin{aligned}
& \kappa^{2} p_{\phi}=\kappa^{2} p(\phi, X)=-\frac{3}{(d r / d z)^{2}}-2(1+z) \frac{d^{2} r / d z^{2}}{(d r / d z)^{3}} \\
& \kappa^{2} \rho_{\phi}=\kappa^{2}\left(2 X \frac{\partial p}{\partial X}-p\right)=\frac{3}{(d r / d z)^{2}}-3 H_{0}^{2} \Omega_{M, 0}(1+z)^{3}
\end{aligned}
$$

From Eq.(5.1), we find that $p(\phi, X)$ is written as a function of $z$. In order to reconstruct $p(\phi, X)$, we further need to rewrite $\phi$ (or $X=\dot{\phi}^{2} / 2$ ) as a function of $z$. One might expect that this may be done using Eq.(5.2). Indeed, it is possible for the case of the quintessence field with a canonical kinetic term and a potential term, where $p(\phi, X)=X-V(\phi)$ and $\rho(\phi, X)=X+V(\phi)$. By summing up Eqs.(5.1) and (5.2), $X$ can be described as a function of $z$. Integrating $X$ with respect to $z$ enables us to rewrite $\phi$ as a function of $z$. Then, by subtracting Eq.(5.1) from Eq.(5.2), $V(\phi)$ can be described as a function of $z$, that is, $\phi$. However, in our case, $p(\phi, X)$ is an arbitrary function of both $\phi$ and $X$ so that it is impossible to rewrite $\phi$ or $X$ as a function of $z$ without identifing the combination of $\phi$ and $X$ in $p(\phi, X)$. p

Of cource, once one specifies the functional form of $p(\phi, X)$, the reconstruction is possible like the case of the quintessence field with a canonical kinetic term and a potential term. For example, for the case of our model Eq.(3.3), we can write $f(\phi)$ and $X$ in terms of observable

\footnotetext{
${ }^{4}$ It is even possible to start from zero energy density with a fine-tuning of the initial conditions.

${ }^{5}$ We also note that the reconstruction method fails if the scalar field has a multi component or is non-minimally coupled [15,16] to the curvature.
} 
quantities in the following manner: Take the ratio of Eq.(5.2) to Eq.(5.1). Then $X$ is written as a function of $z$. Integrating $X$ with respect to $z, \phi$ can be written as a function of $z$. Once again using Eq.(5.1) together with the results obtained, we can rewrite $f$ as a function of $z$. Hence we can reconstruct $f, f(\phi)=f(\phi(z))$.

\section{SUMMARY}

We have shown that a scalar field with only non-canonical kinetic terms can, without the help of potential terms, behave like quintessence or phantom energy component.

We have presented a kinetic counterpart of the Ratra-Peebles model and investigated the structure of the phase plane and shown that the quintessential solution is a late-time attractor. The phase area of the initial conditions for the scalar field which converge on the quintessential solution is smaller than that of the Ratra-Peebles model since there are a trivial solution as well as the scaling solutions in the kinetic Lagrangian. However, for very wide ranges of initial energy density, these energy components converge on common evolutionary tracks.

We have also given a model of a power-law "phantom" field with arbitrary $w$ of $w<-1$ which is stable against perturbation, and have shown that the phantom solution is a latetime attractor. In our model of the phantom field, the initial conditions have to be set carefully because there are a number of scaling solutions. Moreover, the initial energy density should be chosen to be below the present energy scale because the decaying lambda region corresponds to the unstable region. Therefore, there remains a fine-tuning problem of the initial conditions, which is no more severer than that of the cosmological constant. The situation may be improved by including potential terms or by introducing additional fields.

The violation of the weak energy condition is required to construct wormholes [26]. Our model of a phantom field may be used to explore such a fascinating possibility.

\section{ACKNOWLEDGMENTS}

We would like to thank Professor Masahiro Kawasaki and Professor Takashi Nakamura for useful comments. T.C. is also grateful to Dr. Ken-ichi Nakao for critical comments. T.O. would like to thank Professor Yasushi Suto for encouragement. This work was supported in part by JSPS Fellowship for Young Scientists under grant No.3596 (TC) and No.4558 (MY). 


\section{REFERENCES}

[1] J.P. Ostriker and P.J. Steinhardt, Nature 377, 600 (1995); L. Wang, R.R. Caldwell, J.P. Ostriker, and P.J. Steinhardt, astro-ph/9901388.

[2] L.M. Krauss and M.S Turner, Gen. Rel. Grav. 27, 1137 (1995).

[3] S. Perlmutter et al., ApJ. 517, 565 (1999).

[4] B.P. Schmidt et al., ApJ. 507, 46 (1998); A.G. Riess et al., Astron. J. 116, 1009 (1998).

[5] A.D. Dolgov, in The Very Early Universe, edited by G.W.Gibbons, S.W.Hawking, and S.T.Siklos (Cambridge University Press, Cambridge, 1983).

[6] M. Özer and M.O. Taha, Phys. Lett. 171B, 363 (1986); K. Freese, F.C. Adams, J.A. Frieman, and E. Mottola, Nucl. Phys. B287, 797 (1987).

[7] B. Ratra and P.J.E. Peebles, Phys. Rev. D 37, 3406 (1988); P.J.E. Peebles and B. Ratra, Astrophys. J. 325, L17.

[8] Y. Fujii and T. Nishioka, Phys. Rev. D 42, 361 (1990).

[9] J.A. Frieman, C.T. Hill, and R. Watkins, Phys. Rev. D 46, 1226 (1992).

[10] T. Chiba, N. Sugiyama, and T. Nakamura, Mon. Not. Roy. Astron. Soc. 289, L5 (1997); T. Chiba, N. Sugiyama, and T. Nakamura, Mon. Not. Roy. Astron. Soc. 301, 72 (1998).

[11] M.S. Turner and M. White, Phys. Rev. D 56, 4439 (1997).

[12] R.R. Caldwell, R. Dave, and P.J. Steinhardt, Phys. Rev. Lett. 80, 1582 (1998).

[13] I. Zlatev, L. Wang, and P.J. Steinhardt, Phys. Rev. Lett. 82, 896 (1999).

[14] M. Bucher and D. Spergel, Phys. Rev. D 60, 043505 (1999); R.A. Battye, M. Bucher and D. Spergel, astro-ph/9908047.

[15] T. Chiba, Phys. Rev. D 60, 083508 (1999).

[16] F. Perrotta, C. Baccigalupi, and S. Matarrese, Phys.Rev.D 61, 023507 (1999).

[17] P. Binétruy, Phys. Rev. D 60, 063502 (1999); A. Masiero, M. Pietroni, and F. Rosati, hep-ph/9905346.

[18] A. Albrecht and C. Skordis, astro-ph/9908085; V. Sahni and L. Wang, astro-ph/9910097;

T. Barreiro, E.J. Copeland, and N.J. Nunes, astro-ph/9910214.

[19] R.R. Caldwell, astro-ph/9908168.

[20] C. Armendáriz-Picón, T. Damour and V. Mukhanov, Phys. Lett. B458, 209 (1999).

[21] T. Damour and A.M. Polyakov, Nucl. Phys. B423, 532 (1994).

[22] C.M. Will, Theory and experiment in gravitational physics, (Rev.Ed., Cambridge University Press, Cambridge, 1993); C.M. Will, gr-qc/9811036.

[23] T. Nakamura and T. Chiba, Mon. Not. Roy. Astron. Soc. 306, 696 (1999).

[24] A. Starobinsky, JETP Lett. 68, 757 (1998); D. Huterer and M.S. Turner, Phys. Rev. D 60, 081301 (1999); T.D. Saini, S. Raychaudhury, V. Sahni, and A.A. Starobinsky, astro-ph/9910231.

[25] A.R. Liddle and R.J. Scherrer, Phys. Rev. D 59, 023509 (1999).

[26] F.J. Tipler, Phys. Rev. Lett. 37, 879 (1976); M.S. Morris, K.S. Thorne, and U. Yurtsever, Phys. Rev. Lett. 61, 1446 (1988). 


\section{FIGURES}

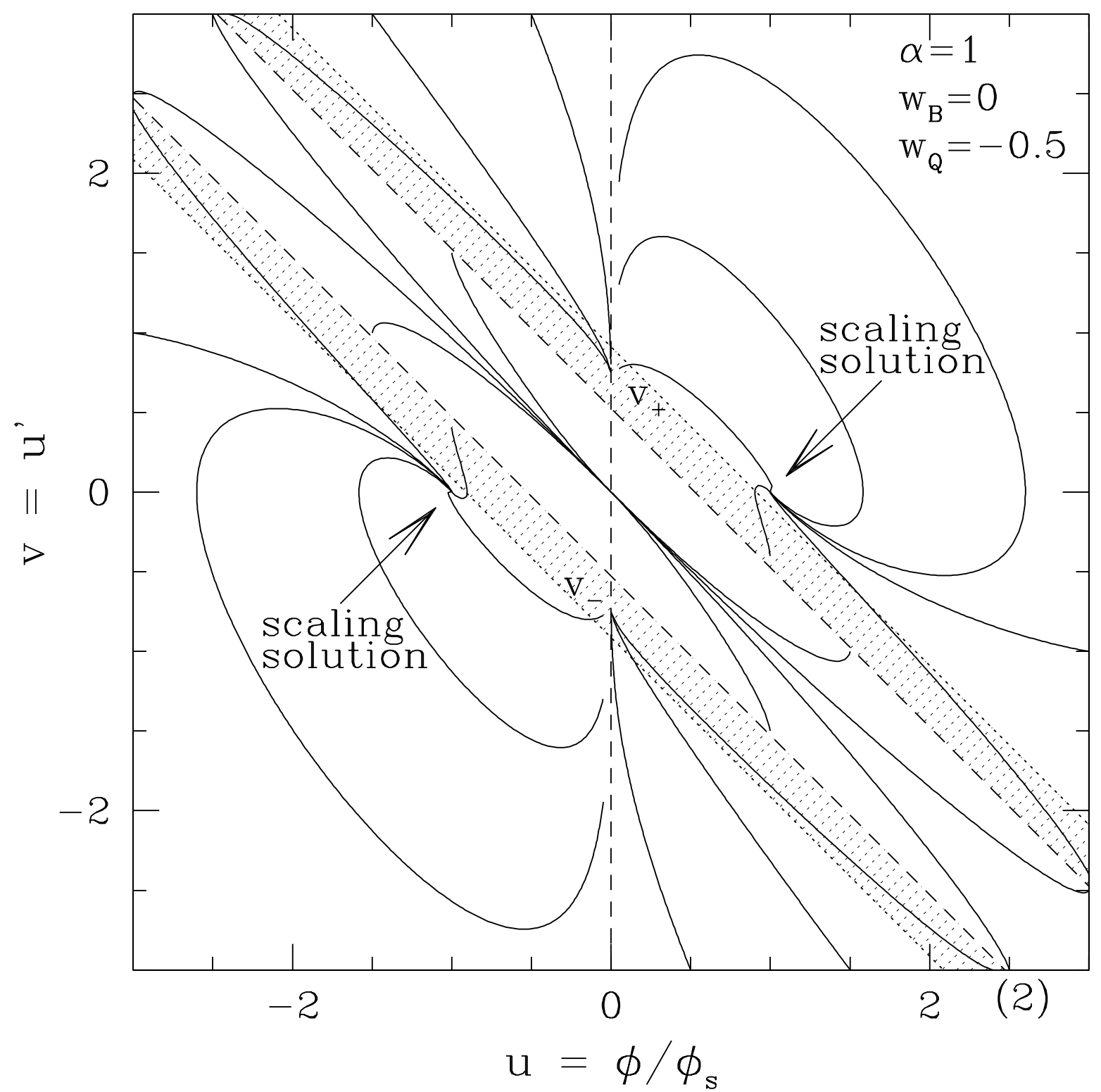

(1)

FIG. 1. Phase plane of the quintessence field in the matter dominated universe. Trajectories in the region between line (1) and (2) converge on the trivial solution $(u, v)=(0,0)$. In the region above line (1) and $u>0$ they converge on the scaling solution $(1,0)$ directly, while in the region below line (2) and $u>0$ they approach and pass through the point $\left(0, v_{-}\right)$, then converge on another scaling solution $(-1,0)$. The speed of sound is imaginary in the shaded region. 


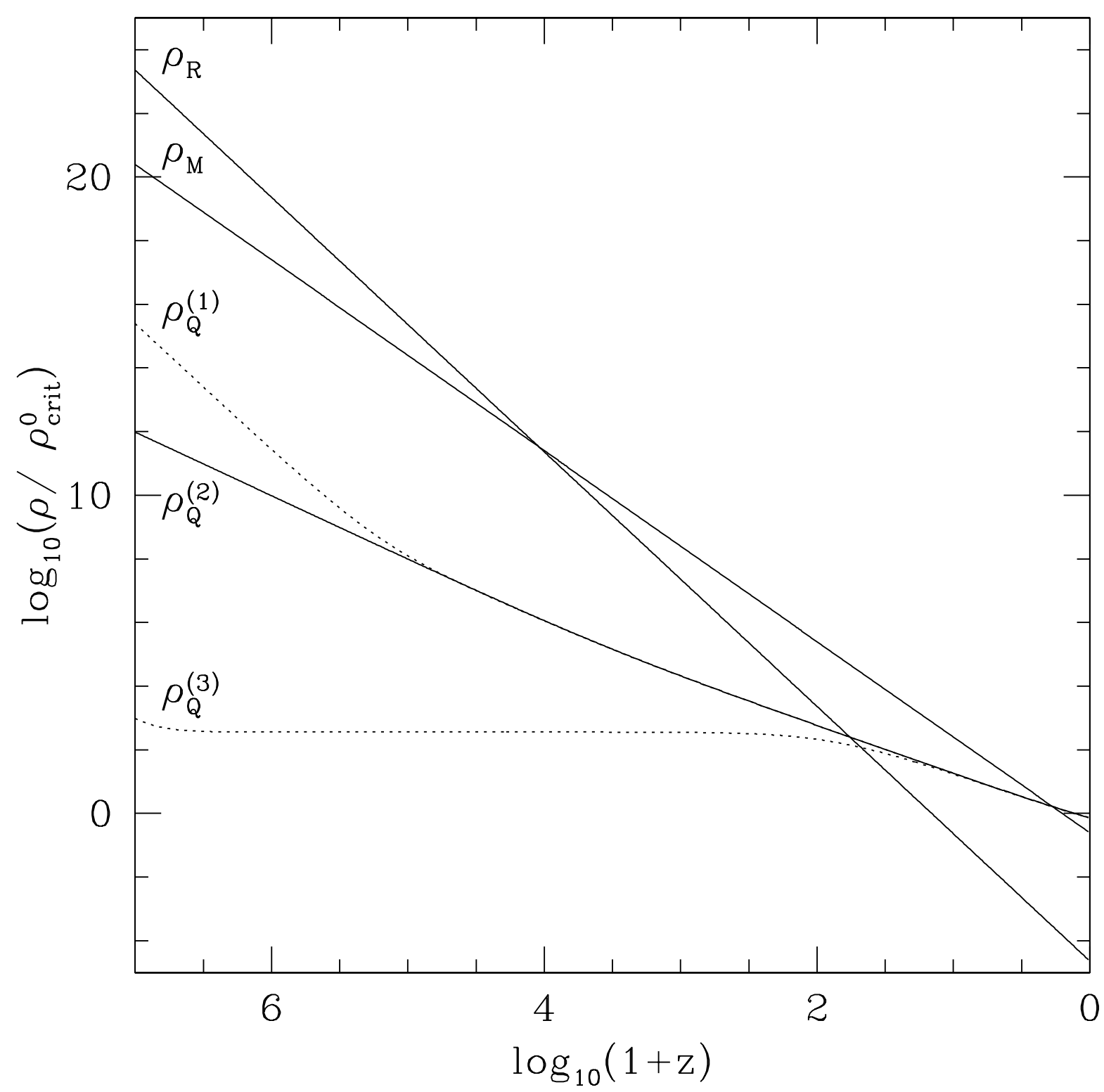

FIG. 2. Energy densities of radiation, $\rho_{R}$, matter, $\rho_{M}$, and quintessence field for various initial conditions, $\rho_{Q}^{(i)}, i=1,2,3$, against the redshift. The initial conditions for $\rho_{Q}^{(2)}$ correspond to that of the scaling solution in the radiation dominated universe. The initial energy density of the quintessence field which converges on the attractor solution spans more than 10 orders of magnitude. 


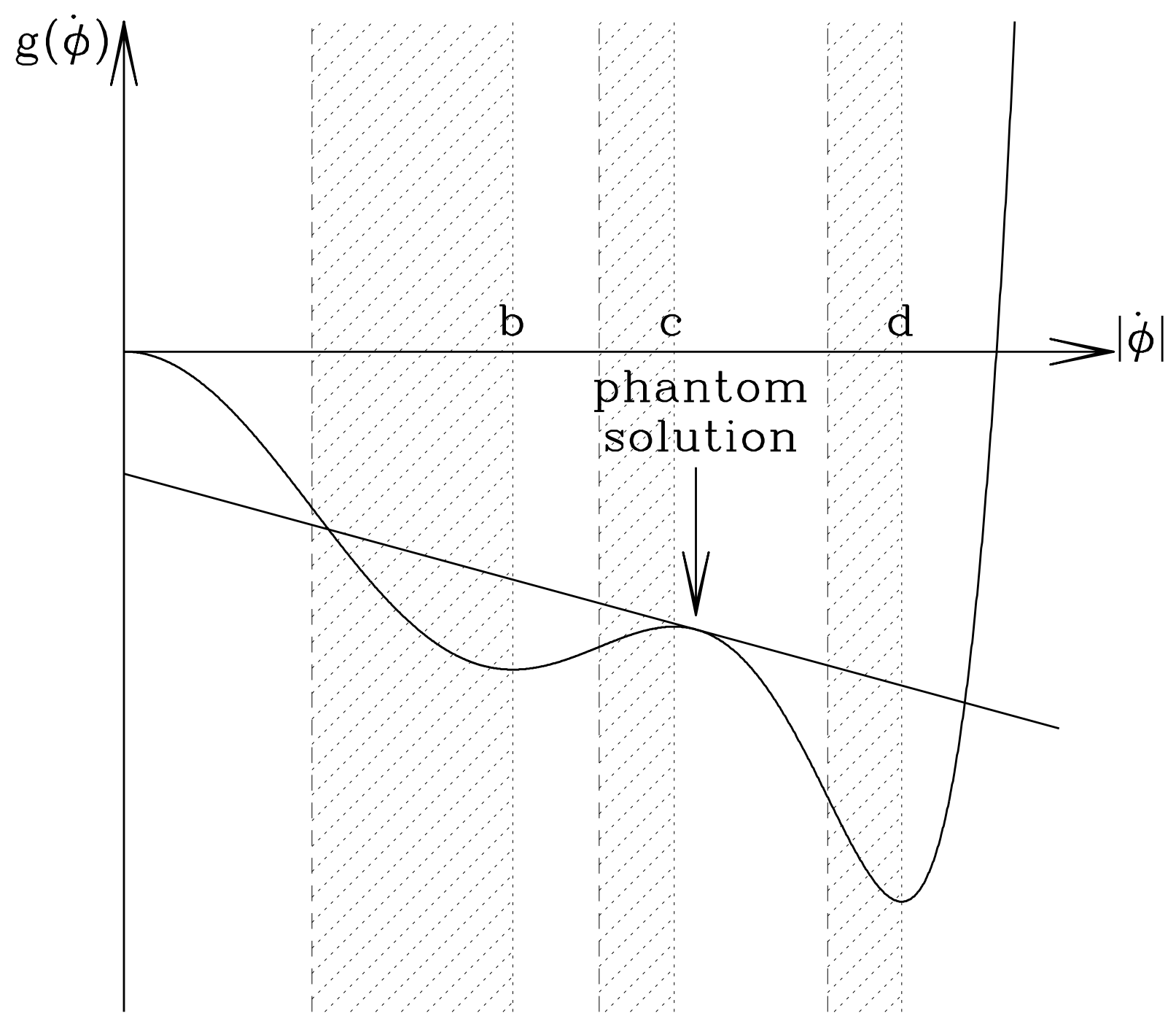

FIG. 3. A sketch of the function $g(\dot{\phi}) . c_{s}^{2}<0$ in the shaded regions. The phantom solution is one of the solutions of Eq.(4.3) at which $\dot{\phi} g_{, \dot{\phi}}<0$ and $g_{, \dot{\phi}} / \dot{\phi} g_{, \dot{\phi} \dot{\phi}}>0$ and the intersection of the tangent with the vertical axis is negative. 


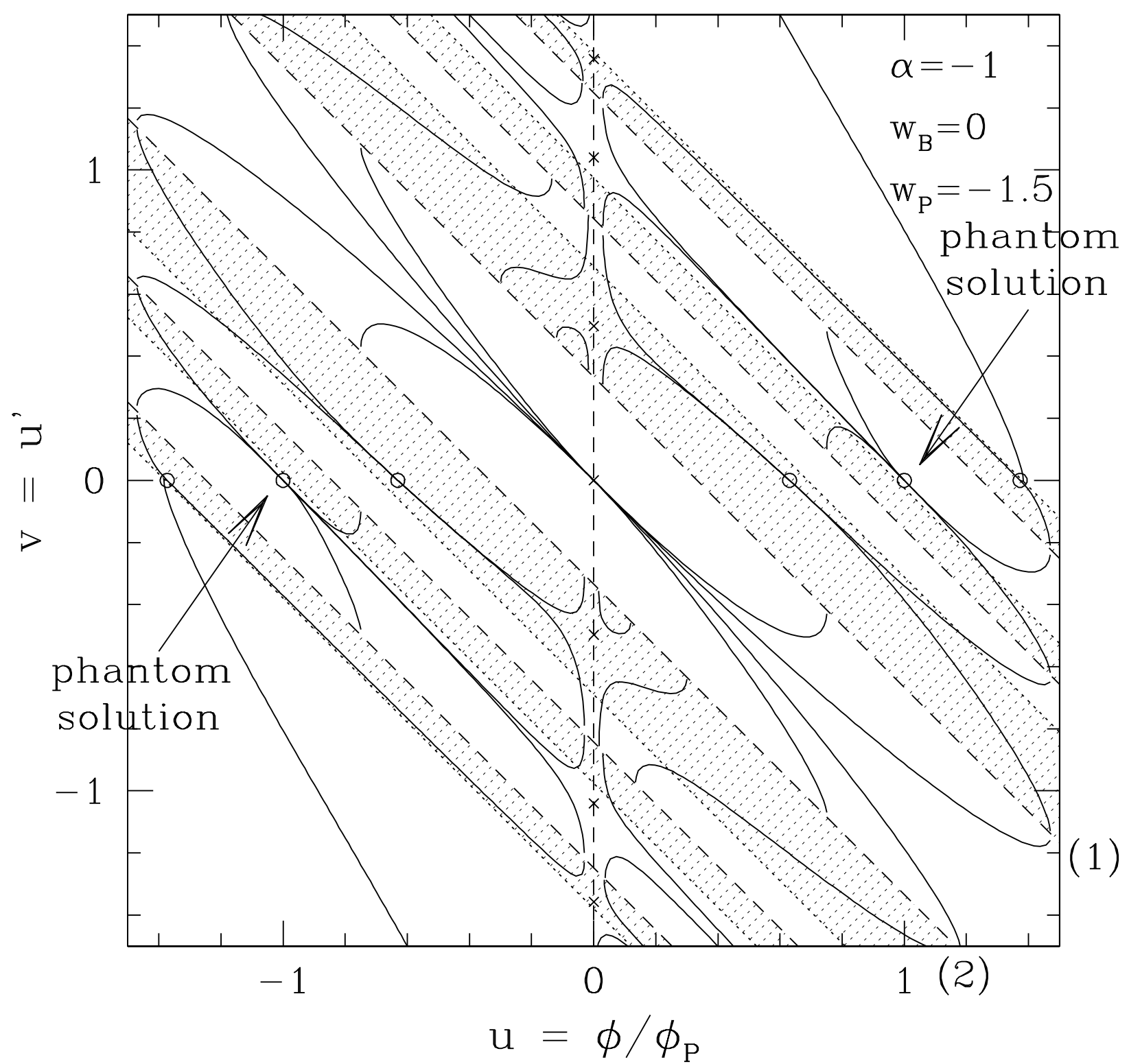

FIG. 4. Phase plane of the phantom field in the matter dominated universe. We set $b^{2}=1 / 2$, $c^{2}=1$ and $d^{2}=2$. The scaling solutions are represented by open circle. Eq.(4.7) is singular on the dashed lines except for the points represented by cross. In the shaded regions corresponding to those in FIG.3, $c_{s}^{2}<0$. Trajectories in the region between line (1) and (2) converge on the trivial solution. In the region above line (1) and $u>0$, they converge on the scaling solutions. In the region below line (2) and $u>0$, trajectory approaches a point on the boundary asymptotically. 


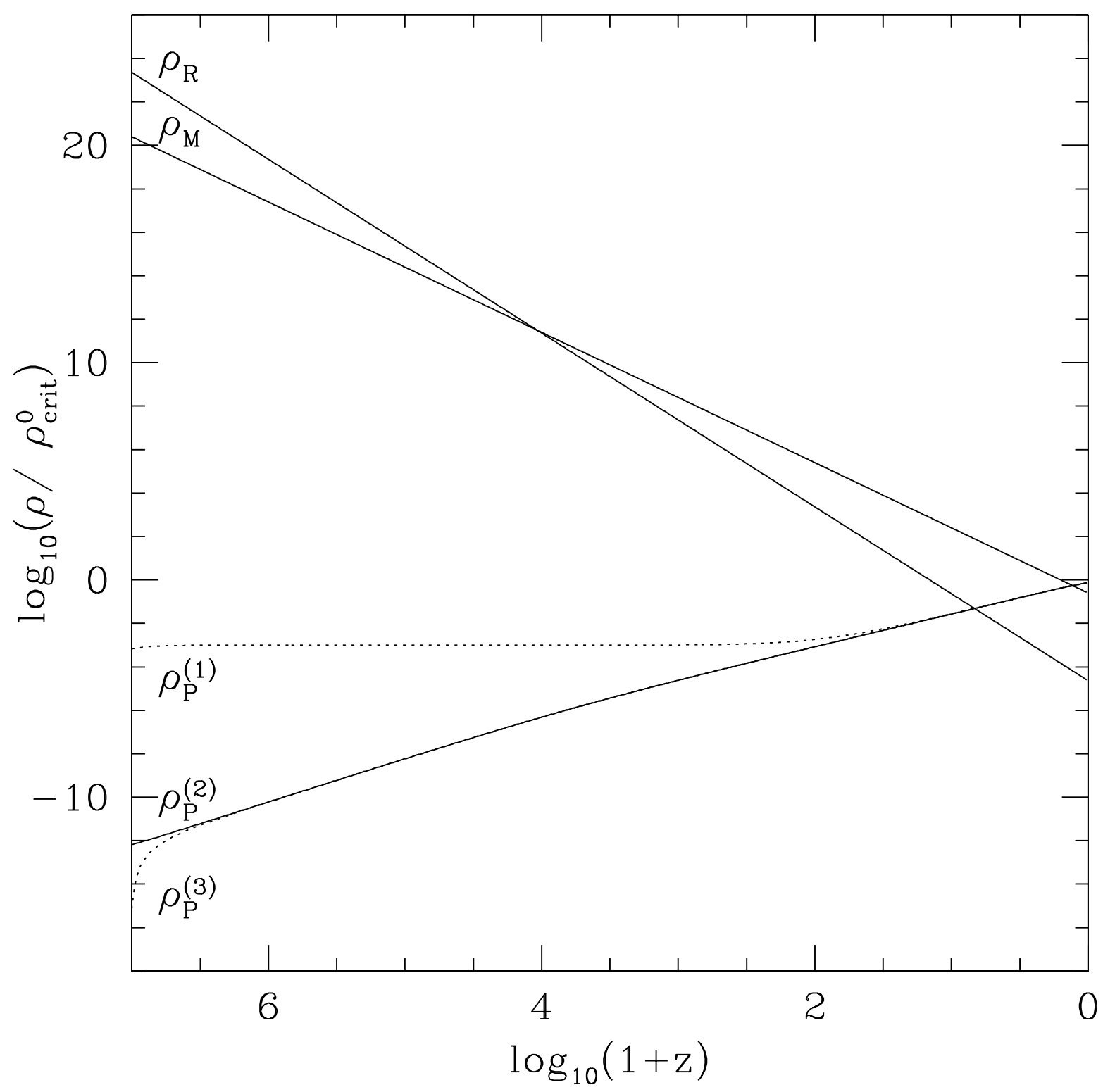

FIG. 5. Energy densities of radiation, $\rho_{R}$, matter, $\rho_{M}$, and phantom field for various initial conditions, $\rho_{P}^{(i)}, i=1,2,3$, against the redshift. The initial conditions for $\rho_{P}^{(2)}$ correspond to that of the phantom solution in the radiation dominated universe. The initial energy density of the phantom field which converges on the phantom solution spans more than 10 orders of magnitude. 